\title{
Stability, Chaos Diagnose and Adaptive Control of Two Dimensional Discrete - Time Dynamical System
}

\author{
Maysoon M. Aziz, Omar M. Jihad \\ Department of Mathematics, College of Computer Science and Mathematics, University of Mosul, Mosul, Iraq \\ Email: aziz_maysoon@yahoo.com,omarmoh1992h@gmail.com,aziz_maysoon@uomosul.edu.iq
}

How to cite this paper: Aziz, M.M. and Jihad, O.M. (2021) Stability, Chaos Diagnose and Adaptive Control of Two Dimensional Discrete - Time Dynamical System. Open Access Library Journal, 8: e7270. https://doi.org/10.4236/oalib.1107270

Received: February 27, 2021

Accepted: March 23, 2021

Published: March 26, 2021

Copyright $\odot 2021$ by author(s) and Open Access Library Inc.

This work is licensed under the Creative Commons Attribution International License (CC BY 4.0).

http://creativecommons.org/licenses/by/4.0/

\section{(c) (i) Open Access}

\begin{abstract}
In this paper $2 \mathrm{D}$ discrete time dynamical system is presented. The fixed points were found. The stability of fixed points is measured by characteristic roots, jury criteria, Lyapunov function. All show that the system is unstable, and analyzing the dynamic behavior of the system finds bifurcation diagrams at the bifurcation parameter. Newton's Raphson numerical method was used the roots of the system with the minimum error. Then, chaoticity is measured by the phase space; maximum Lyapunov exponent is obtain as ( $\left.L_{\max }=2.394569\right)$; Lyapunov dimension is obtain as $\left(D_{L}=3.366413\right)$; binary test $(0-1)$ is obtain as $(k=0.982)$. All show that the system is chaotic. Finally, the adaptive control was performed. Moreover, theoretical and graphical results of the system after control show the system is stable and Lyapunov exponent is obtained as: $L_{1}=-0.390000, L_{2}=-0.500000$, so the system is regular.
\end{abstract}

\section{Subject Areas \\ Dynamical System}

\section{Keywords}

Stability, Jury Criteria, Lyapunov Function, Chaotic System, Lyapunov Exponent, Binary Test, Adaptive Control

\section{Introduction}

In the last two decades, the interest in dynamical systems has increased, because they are an important concept in describing the behavior of many models and in various fields. Some studies and research have focused on discrete dynamical systems in which the systems are described in the form of difference equations 
[1], as intermittent dynamic systems are more appropriate in describing models, especially when Models have non-overlapping generations, as the computational techniques used in discrete dynamical systems are more effective for numerical simulations, unlike in continuous dynamic systems. Mathematical models have been studied in biology and the environment, which have been described in the form of food chains. Food chains is one of the basic relationships that describe for us the interactions that occur between societies in the field of ecology, which studies the interrelationships between living organisms and the environment in which they are. Malthus [2] presented a study on the interactions that occur in societies that consist of one species, which were described using difference mathematical equation. There are many mathematical models in ecology that have been touched upon by researchers in this field. One of the most important mathematical models that have received wide attention in recent years is the prey and predator model due to its wide scope of application. The interaction between the prey community and the predator was described by Volterra [3], Lutka [4]. This type of model was found in temperate regions due to its appropriate environment seasonality, as Holling presented [5] a more realistic model of prey predator describing for us the interaction of three types of societies, as the relationship in the intermittent dynamic systems between prey and predator societies was and remains one of the important topics in many studies and research because of its importance. This type of the models depends on many assumptions, including that prey societies grow in a limited way when the predator community is absent, and predation societies depend on the presence of prey in order to survive, that is, the rate of the prey community is proportional to the predator community, and finally, we can say that the models in the environmental science are not simple and we cannot rely on the results obtained as stable and perfect results.

Accordingly, the research was arranged as follows: system description, system analysis and finding fixed points [6], stability analysis [7] [8] [9] of the fixed points by (characteristic roots equation, jury criteria [10], Lyapunov function [10]), numerical behavior study and finding diagrams of bifurcation of the system [11] [12], chaos analysis using maximum Lyapunov exponent and the Lyapunov dimension [13] and the binary test $(0-1)$ [14]-[20]. In the final section, adaptive control [21] [22] of the chaotic system is performed, and stability tests and the Lyapunov exponent test are performed.

\section{System Description}

In this work, a two-dimensional discrete time dynamical system was taken [23] and defined as follows:

$$
\begin{gathered}
\left\{\begin{array}{c}
x_{t+1}=x_{t}+a_{1} x_{t}\left(1-x_{t}\right)-a_{2} x_{t} y_{t} \\
y_{t+1}=y_{t}+a_{2} y_{t}\left(x_{t}-y_{t}\right)
\end{array}\right. \\
a_{1}=2.4, a_{2}=2
\end{gathered}
$$


where $x_{t}$ represents the prey society and $y_{t}$ represents the predator community in discrete time $(t)$, and that $a_{1}, a_{2}$ represent the parameters of the system and are positive, the part: $x_{t}\left[1+a_{1}\left(1-x_{t}\right)\right]$ is represents the rate of increase of the prey community in the absence of the predation community, and that Part $a_{2} x_{t} y_{t}$ represents the rate of decline of the prey community due to the presence of the predation community, and $a_{2}$ represents the predation parameter, while the part $y_{t}\left[1+a_{2}\left(x_{t}-y_{t}\right)\right]$ represents the variance in the size of the predation population which depends on the size of the prey community.

\section{System Analysis}

\subsection{Jacobian Matrix}

For 2D discrete dynamical system (1) with continuous differentiable transition function $g_{1}$ and $g_{2}$, given by

$$
\begin{aligned}
& x_{t+1}=g_{1}\left(x_{t}, y_{t}\right) \\
& y_{t+1}=g_{2}\left(x_{t}, y_{t}\right)
\end{aligned}
$$

The Jacobian Matrix of system (1) is:

$$
J_{\left(x_{t}, y_{t}\right)}=\left[\begin{array}{ll}
\frac{\partial g_{1}}{\partial x_{t}} & \frac{\partial g_{1}}{\partial y_{t}} \\
\frac{\partial g_{2}}{\partial x_{t}} & \frac{\partial g_{2}}{\partial y_{t}}
\end{array}\right]=\left[\begin{array}{cc}
1+a_{1}-2 a_{1} x_{t}-a_{2} y_{t} & -a_{2} x_{t} \\
a_{2} y_{t} & 1+a_{2} x_{t}-2 a_{2} y_{t}
\end{array}\right]
$$

\subsection{Fixed Point}

In this section we find the fixed points of system (1), assume that $g_{1}\left(x_{t}, y_{t}\right)=x_{t}$, $g_{2}\left(x_{t}, y_{t}\right)=y_{t}$

$$
\begin{gathered}
x_{t}=x_{t}+a_{1} x_{t}\left(1-x_{t}\right)-a_{2} x_{t} y_{t} \\
y_{t}=y_{t}+a_{2} y_{t}\left(x_{t}-y_{t}\right)
\end{gathered}
$$

from Equations (4) and (5) we get the following fixed points:

$$
q_{0}=(0,0), \quad q_{1}=(1,0), \quad q_{2}=\left(\frac{a_{1}}{a_{1}+a_{2}}, \frac{a_{1}}{a_{1}+a_{2}}\right)
$$

Theorem (1): Let

$$
G(\lambda)=a_{2} \lambda^{2}+a_{1} \lambda+a_{0}=0
$$

A characteristic equation of (3), the following cases are true:

1) If the absolute value of the roots of Equation (6) is less than one, then the fixed point of the system (1) is locally asymptotically stable and is called the sink.

2) If the absolute value of the roots of Equation (6) is greater than one, then the fixed point of the system (1) is unstable and is usually called the source, but if at least one of the values of the roots of Equation (6) is greater than one, then the fixed point is called the Saddle.

3) If the absolute value of the roots of Equation (6) is equal to one, then the fixed point of the system (1) is called the non-hyperbolic point. But if there are 
no roots of values equal to one, then the fixed point is called the hyperbolic point.

\section{Stability Analysis}

In this section, the stability of the fixed points of the system (1) using the following criteria:

\subsection{Characteristic Equation Roots}

Substituting the point $q_{0}$ into (3) we get:

$$
\begin{gathered}
J_{(0,0)}=\left[\begin{array}{cc}
1+a_{1} & 0 \\
0 & 1
\end{array}\right] \\
\operatorname{det}(\lambda I-J)=0 \\
\left|\begin{array}{cc}
\lambda-1-a_{1} & 0 \\
0 & \lambda-1
\end{array}\right|=0
\end{gathered}
$$

And from Equation (2) we get:

$$
\lambda^{2}-4.4 \lambda+3.4=0
$$

so the roots of quadratic Equation (7) are: $\lambda_{1}=1, \lambda_{2}=3.4$

since $\left|\lambda_{1}\right|=1,\left|\lambda_{2}\right|=3.4$, so by theorem (1) we get system (1) is unstable at $q_{0}$, similarly we test the points $q_{1}$ and $q_{2}$, the results shown in Table 1 that the three points $q_{0}, q_{1}, q_{2}$ are saddle.

Lemma (1):

Let the characteristic equation of system (1)

$$
G(\lambda)=a_{2} \lambda^{2}+a_{1} \lambda+a_{0}=0
$$

Then it's jury Table 2 is:

Table 1. Stability of fixed points by characteristic equation.

\begin{tabular}{ccc}
\hline Fixed points & Characteristic Equation Roots & the description \\
\hline$q_{0}=(0,0)$ & $\lambda_{1}=1, \lambda_{2}=3.4$ & unstable \\
$q_{1}=(1,0)$ & $\lambda_{1}=2.2283, \lambda_{2}=0.6283$ & unstable \\
$q_{2}=(0.5454,0.5454)$ & $\lambda_{1,2}=1.2907$ & unstable \\
\hline
\end{tabular}

Table 2. Jury table.

\begin{tabular}{ccc}
\hline$\lambda^{0}$ & $\lambda^{1}$ & $\lambda^{2}$ \\
\hline$a_{0}$ & $a_{1}$ & $a_{2}$ \\
$a_{2}$ & $a_{1}$ & $a_{0}$ \\
$b_{0}$ & $b_{1}$ & \\
$b_{1}$ & $b_{0}$ & \\
$c_{0}$ & & \\
\hline
\end{tabular}


Such that

$$
\begin{aligned}
b_{k} & =\left|\begin{array}{cc}
a_{0} & a_{n-k} \\
a_{n} & a_{k}
\end{array}\right|, k=0,1, \quad n=2 \\
c_{k} & =\left|\begin{array}{cc}
b_{0} & b_{n-1-k} \\
b_{n-1} & b_{k}
\end{array}\right|, k=0, \quad n=2
\end{aligned}
$$

We say that the fixed point of the system (1) is stable if the satisfies following conditions:

$$
\begin{gathered}
G(1)>0 \\
(-1)^{n} G(-1)>0 \\
\left|a_{0}\right|<a_{n},\left|b_{0}\right|>\left|b_{n-1}\right|,\left|c_{0}\right|>\left|c_{n-2}\right|
\end{gathered}
$$

Otherwise, fixed points are unstable.

\subsection{Jury Stability Criteria}

We test the stability of point $q_{0}$, by using lemma (1) and values from eq. (7) we get the jury Table 3 , since the condition $\left|a_{0}\right|=3.4>a_{2}=1$, so the point $q_{0}$ is unstable. Similarly, we test the rest of the points $q_{1}, q_{2}$, which shows that they are unstable, so the system (1) is unstable.

\subsection{Lyapunov Function Criteria}

The fixed points are said to be stable using the criterion of the Lyapunov function if $\Delta V \leq 0$ except for the origin, which is stable, and to study the stability of the fixed points of the system (1) we impose the quadratic equation of the following Lyapunov function:

$$
V(x, y)=x^{2}+y^{2}>0
$$

Where $x, y$ are not equal to zero, and using $\Delta V$ we get:

$$
\begin{aligned}
\Delta V\left(x_{t}, y_{t}\right)= & V\left(x_{t+1}, y_{t+1}\right)^{2}-V\left(x_{t}, y_{t}\right)^{2} \\
= & \left(x_{t}+a_{1} x_{t}\left(1-x_{t}\right)-a_{2} x_{t} y_{t}\right)^{2}+\left(y_{t}+a_{2} y_{t}\left(x_{t}-y_{t}\right)\right)^{2} \\
& -\left(x_{t}\right)^{2}-\left(y_{t}\right)^{2}
\end{aligned}
$$

By substituting (2) in Equation (8) and test the point $q_{2}=(0.5454,0.5454)$ we get:

Table 3. Jury table of the point $q_{0}$.

\begin{tabular}{ccc}
\hline$\lambda^{0}$ & $\lambda^{1}$ & $\lambda^{2}$ \\
\hline 3.4 & -4.4 & 1 \\
1 & -4.4 & 3.4 \\
10.56 & -10.56 & \\
-10.56 & 10.56 & \\
0 & & \\
\hline
\end{tabular}




$$
\begin{aligned}
\Delta V(0.5454,0.5454) & \\
= & (0.5454+2.4 \times 0.5454 \times(1-0.5454)-2 \times 0.5454 \times 0.5454)^{2} \\
& +(0.5454+2 \times 0.5454 \times(0.5454-0.5454))^{2}-(0.5454)^{2}-(0.5454)^{2} \\
= & 0.1438>0
\end{aligned}
$$

It is clear that $V$ is positive definite and $\Delta V$ is positive, hence the fixed point $q_{2}$ is unstable and so system (1) is unstable.

\section{The Graphical and Numerical Behavior of System (1)}

\subsection{Newtons' - Raphson Method}

Newton's Raphson method is one of the important numerical methods for finding the roots of the difference equations. By using a written programme on MATLAB we get the best result obtained for the system $(1)$ is $(x, y)=(0.5,0.5)$ with minimum error (0.0001).

\subsection{Trajectories of System (1)}

In this section the time behavior of the system (1) was studied and the parameters were fixed at the values $a_{1}=2.4, a_{2}=2$ with the values $(x, y)=(0.5,0.5)$ and for (1000) iterations, shown in Figure 1. It turns out that the system behaves unstable.

\subsection{Phase Space of System (1)}

In this section, the phase space of the system (1) was found, the parameters were fixed at the values $a_{1}=2.4, a_{2}=2$ with the values $(x, y)=(0.5,0.5)$ and shown in Figure 2 which show that the system behaves chaotic. In Figure 3
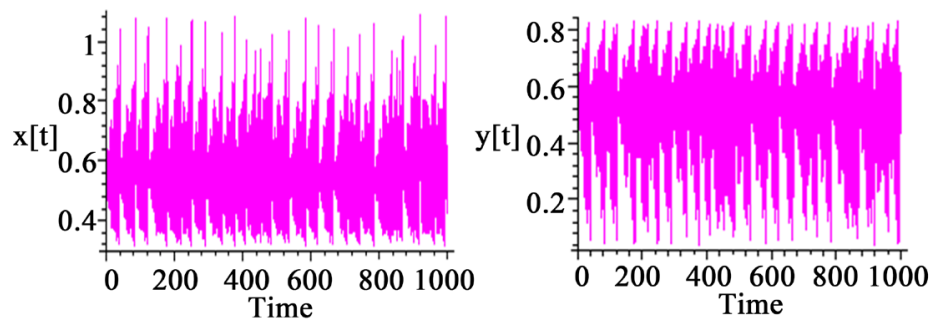

Figure 1. The trajectories of state variables $x_{t}$ and $y_{t}$, when $a_{1}=2.4$.

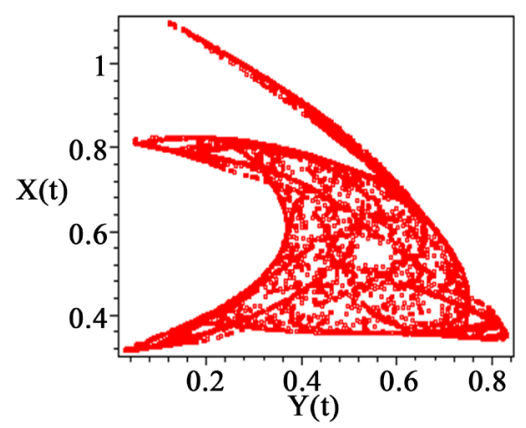

Figure 2. The phase space of the system (1) at parameter $a_{1}=2.4$. 

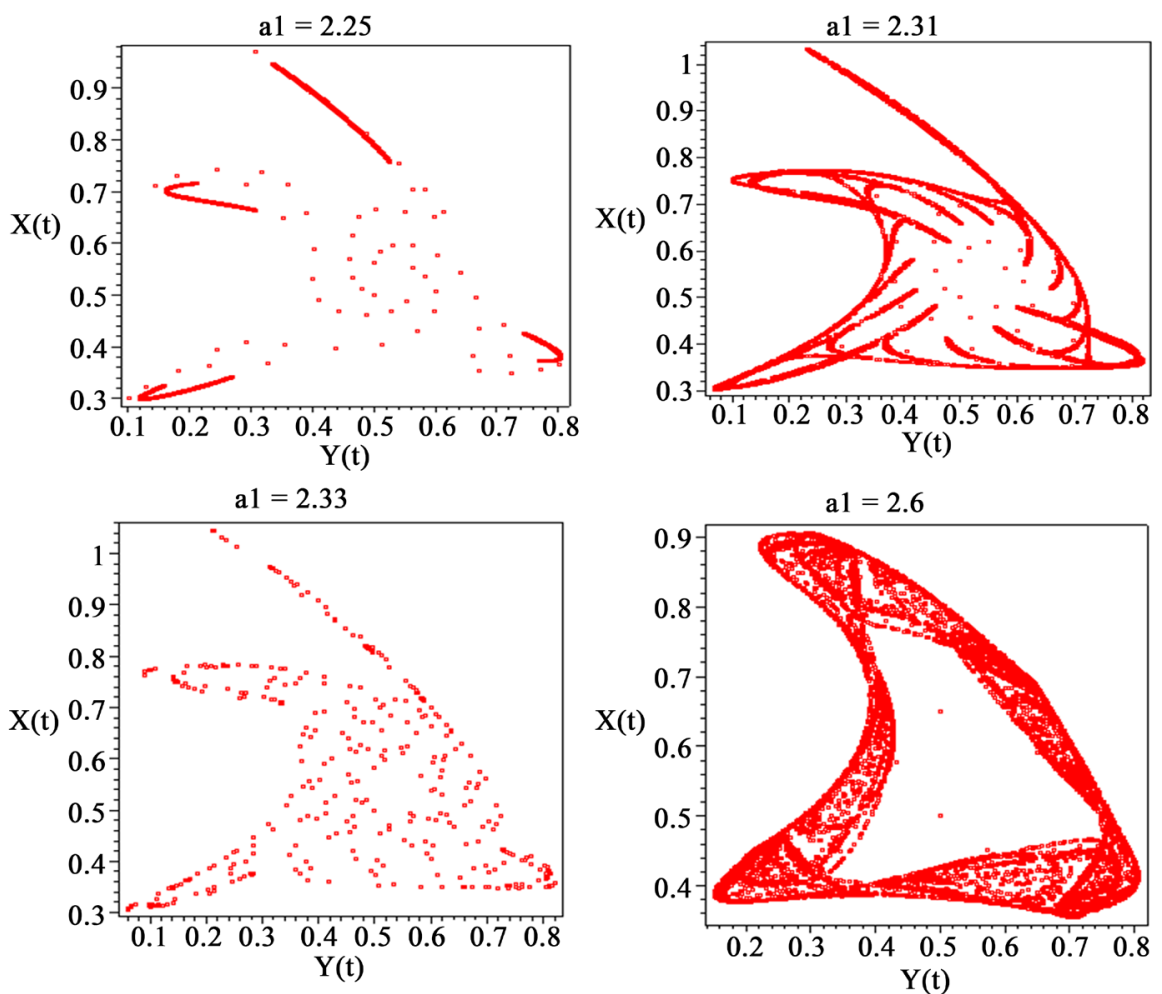

Figure 3. The phase space of the system (1) with some values at parameter $a_{1}$.
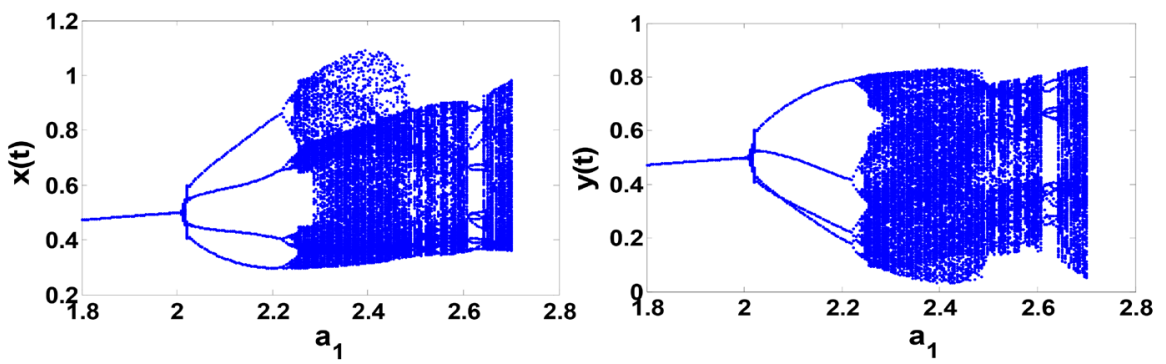

Figure 4. Bifurcation of parameter $a_{1}$ with variable $x_{t}, y_{t}$.

shows that the system (1) generates trajectories chaotic for $x_{t}$ and $y_{t}$ at some values parameter $a_{1}$.

\subsection{Bifurcation Diagram}

In this section the bifurcation diagrams of system (1) are found at the bifurcation parameter $a_{1}$, the parameters $a_{2}=2$ with the values $(x, y)=(0.5,0.5)$ and the $a_{1}$ parameter ranging from 1.8 to 2.7 showing the behavior chaotic of the system (1), also the internal balance of the parameter $a_{1}$ on the period [2.6, 2.65], as shown in Figure 4.

\section{Lyapunov Exponent and Lyapunov Dimension}

The Lyapunov exponent is one of the important tests in detecting the chaotic behavior of dynamic systems, as the system is said to be a chaotic system if one 
of the values of the Lyapunov exponent is greater than zero, and by using a mathematical program in MATLAB, the Lyapunov exponent of the system (1) was tested and the following values were obtained:

$$
L_{1}=2.394569, L_{2}=-1.011898
$$

Since one of the values of the Lyapunov exponent of system (1) is a positive value, then system is chaotic, show in Figure 5.

To calculate the Lyapunov dimension of the system (1), we use the following law:

$$
D_{L}=p+\frac{L_{1}}{\left|L_{2}\right|}=1+\frac{2.394569}{|1.011898|}=3.366413
$$

\section{Binary Test ( 0 - 1)}

In this section, the binary test was used to analysis the chaos of the system (1), a time series $x_{t}$ was generated from the regularity (1) at the parameters $a_{1}=2.4, a_{2}=2$ and the values $(x, y)=(0.5,0.5)$ For (1000) iterations, and by using a mathematical program in MATLAB, the binary selection of the system (1), we calculate $p_{c}(t)$ with $q_{c}(t)$ for $t=100$ and chose(c) is random value within the period $(0, \pi)$, show in Figure $6(\mathrm{a})$, which shows us the behavior aligned to the system and similar to Brownian movement, and the average square displacement $M_{c}(t)$ was calculated with time $k$, shown in Figure 6(b), which shows us that the average square of the displacement $M_{c}(t)$ It is a function that grows linearly with time $(t)$, and by finding the mean $(k)$ of the contiguous growth $k_{c}$ of the mean of the displacement square $M_{c}(t)$ where $t=$ 100 shows that the system behaves in chaotic behavior $(k=0.982)$ as the mean $(k)$ approaches the one shown in Figure 6(c).

\section{Adaptive Control Technique}

To achieve the stability of the chaotic system (1) we will design an adaptive control law with the unknown parameter $a_{1}$.

$$
\begin{aligned}
& x_{t+1}=x_{t}+a_{1} x_{t}\left(1-x_{t}\right)-2 x_{t} y_{t}+u_{1} \\
& y_{t+1}=y_{t}+2 y_{t}\left(x_{t}-y_{t}\right)+u_{2}
\end{aligned}
$$

where $u_{1}, u_{2}$ are the controllers for the adaptive and are known as follows:

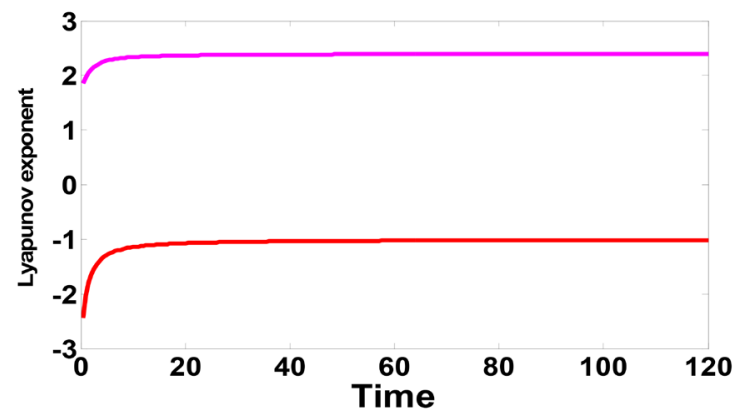

Figure 5. Lyapunov exponent of the system (1). 

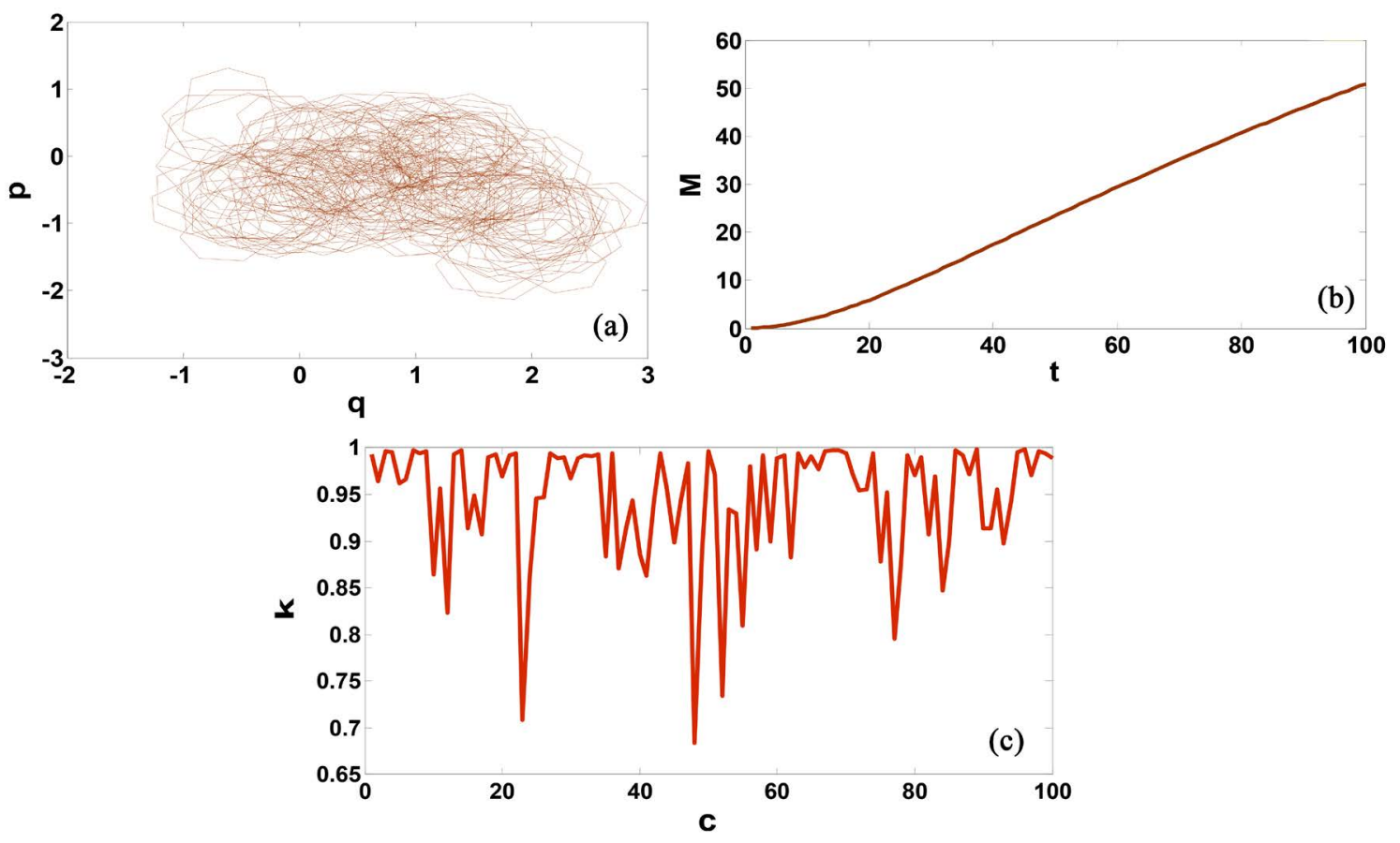

Figure 6. The binary test of system (1) with $a_{1}=2.4$.

$$
\begin{aligned}
& u_{1}=-x_{t}-\hat{a}_{1} x_{t}\left(1-x_{t}\right)+2 x_{t} y_{t}-M_{1} x_{t} \\
& u_{2}=-y_{t}-2 y_{t}\left(x_{t}-y_{t}\right)-M_{2} y_{t}
\end{aligned}
$$

where $M_{1}, M_{2}$ are positive constants and the parameter $\hat{a}_{1}$ is an approximate parameter of the parameter $a_{1}$, and by substituting (10) in (9) we get:

$$
\begin{aligned}
& x_{t+1}=\left(a_{1}-\hat{a}_{1}\right) x_{t}\left(1-x_{t}\right)-M_{1} x_{t} \\
& y_{t+1}=-M_{2} y_{t}
\end{aligned}
$$

Let the error for the discretionary parameter be defined as follows:

$$
e_{a}=a_{1}-\hat{a}_{1}
$$

Substituting (12) into (11) we get:

$$
\begin{aligned}
& x_{t+1}=e_{a} x_{t}\left(1-x_{t}\right)-M_{1} x_{t} \\
& y_{t+1}=-M_{2} y_{t}
\end{aligned}
$$

\subsection{Numerical Results}

In this section, we will test the stability of the fixed points of the system (1) in the controlled system (11) with the values $(x, y)=(0.5,0.5)$ and $M_{1}=0.4$, $M_{2}=0.5$ and the parameter $\hat{a}_{1}$ is an estimated parameter of the parameter $a_{1}$, and let $\hat{a}_{1}=2.39$.

\subsubsection{Characteristic Equation Roots}

From (11) the Jacobian matrix is:

$$
J_{(x, y)}=\left[\begin{array}{cc}
-0.39-0.02 x_{t} & 0 \\
0 & -0.5
\end{array}\right]
$$


Table 4 shows that the testing of the $q_{0}, q_{1}, q_{2}$ of system (11) are stable.

\subsubsection{Jury's Test}

We test the stability of the fixed point $q_{0}=(0,0)$, from characteristic equation $\lambda^{2}+0.89 \lambda+0.195=0$ at point $q_{0}$ we get $a_{0}=0.195, a_{1}=0.89, a_{2}=1$

Then we get Jury Table 5, since, all conditions are satisfies lemma (1), therefore the fixed point $q_{0}$ is stable. Similarly, we test the rest of the points $q_{1}, q_{2}$, shown that all are stable.

\subsubsection{Lyapunov Function Test}

$$
\Delta V\left(x_{t}, y_{t}\right)=\left(x_{t+1}\right)^{2}+\left(y_{t+1}\right)^{2}-\left(x_{t}\right)^{2}-\left(y_{t}\right)^{2}
$$

From the system (11) we get

$$
\Delta V\left(x_{t}, y_{t}\right)=\left(\left(a_{1}-\hat{a}_{1}\right) x_{t}\left(1-x_{t}\right)-M_{1} x_{t}\right)^{2}+\left(-M_{2} y_{t}\right)^{2}-\left(x_{t}\right)^{2}-\left(y_{t}\right)^{2}
$$

Substituting the parameters $\hat{a}_{1}, a_{1}, M_{1}, M_{2}$ we obtain

$$
\Delta V\left(x_{t}, y_{t}\right)=\left(0.01 x_{t}\left(1-x_{t}\right)-0.4 x_{t}\right)^{2}+\left(-0.5 y_{t}\right)^{2}-\left(x_{t}\right)^{2}-\left(y_{t}\right)^{2}
$$

We test the fixed points $q_{0}, q_{1}, q_{2}$, it is clear that $V$ is positive definite and $\Delta V$ is negative definite, consequently the adaptive strategy success to control system (1), and Table 6 illustrates this.

Table 4. Stability of fixed points by characteristic equation for system (11).

\begin{tabular}{ccc}
\hline Fixed points & Characteristic Equation Roots & the description \\
\hline$q_{0}=(0,0)$ & $\lambda_{1}=0.39, \lambda_{2}=0.5$ & stable \\
$q_{1}=(1,0)$ & $\lambda_{1}=0.41, \lambda_{2}=0.5$ & stable \\
$q_{2}=(0.5454,0.5454)$ & $\lambda_{1}=0.4008, \lambda_{2}=0.5$ & stable \\
\hline
\end{tabular}

Table 5. The results Jury test of $q_{0}$.

\begin{tabular}{ccc}
\hline$\lambda^{0}$ & $\lambda^{1}$ & $\lambda^{2}$ \\
\hline 0.195 & 0.89 & 1 \\
1 & 0.89 & 0.195 \\
-0.962 & -0.7165 & \\
-0.7165 & -0.962 & \\
0.4121 & & \\
\hline
\end{tabular}

Table 6. Results of the Lyapunov function test of the system (11).

\begin{tabular}{ccc}
\hline Fixed points & Results of the Lyapunov function test & the description \\
\hline$q_{0}=(0,0)$ & $\Delta V=0$ & stable \\
$q_{1}=(1,0)$ & $\Delta V=-0.84$ & stable \\
$q_{2}=(0.5454,0.5454)$ & $\Delta V=-0.4647$ & stable \\
\hline
\end{tabular}




\subsection{Lyapunov Exponent}

In this section, the Lyapunov exponent test of the system was performed and the values were obtained $L_{1}=-0.390000, L_{2}=-0.500000$, accordingly, the system is regular and Figure 7 shows that.

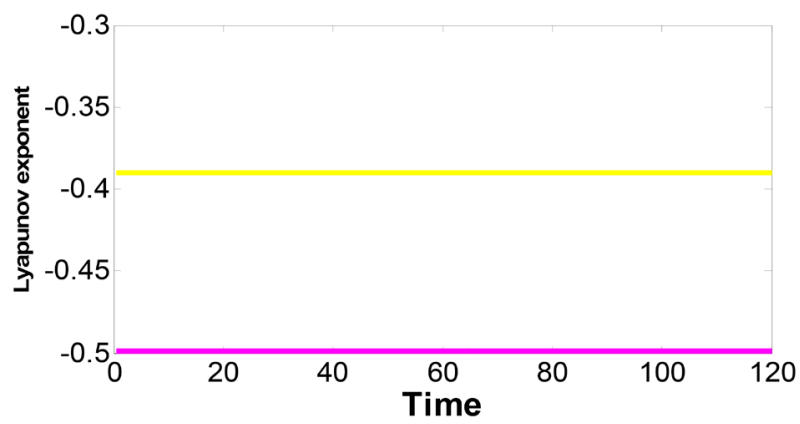

Figure 7. Lyapunov exponent of system (11).

\section{Conclusion}

In this research, 2D discrete - time dynamical system was taken, the system was analyzed, fixed points were found, and the stability analyzed for fixed points using (roots characteristic equation, Jury test, Lyapunov function test). The roots of the system were found using Newton's Raphson numerical method, and the dynamic behavior was analyzed and studied. The phase space of the system shows that the system is unstable at parameter $a_{1}=2.4$. For the chaos analysis of the system, the bifurcation diagrams of the bifurcation parameter $a_{1}=2.4$ are found for the system, and the Lyapunov exponent test was used and the value ( $\left.L_{\max }=2.394569\right)$ was obtained, which is an indication of the chaos of the system. And Lyapunov dimension was calculated as $\left(D_{L}=3.366413\right)$. When using the binary test $(0-1)$, it was found that the value of $(k=0.982)$ with the parameter $a_{1}=2.4$ which is an indication of the chaos of the system. Finally, the adaptive control of the system and the stability test of the system after the control was performed which showed us that the system is stable, and when we tested the Lyapunov exponent $\left(L_{1}=-0.390000, L_{2}=-0.500000\right)$ shown the regular behavior of the system.

\section{Acknowledgements}

We would like sincerely thank and also acknowledge to the university of Mosul and college of computer science and mathematics for the support and encouragement that help us to improve the quality of this work.

\section{Conflicts of Interest}

The authors declare no conflicts of interest regarding the publication of this paper.

\section{References}

[1] Elaydi, S. (2008) An Introduction to Difference Equations. Third Edition, Springer 
International Edition, Berlin.

[2] Malthus, T.R. (1798) An Essay on the Principle of Population. J. Johnson, London.

[3] Voltera, V. (1962) Opere matematiche: Memorie e note. Vol. V, (Cremon) Acc. Naz. dei Lincei, Roma.

[4] Lotka, A.J. (1925) Elements of Physical Biology. Williams \& Wilkins Co., Baltimore.

[5] Holling, C.S. (1965) The Functional Response of Predator to Prey Density and Its Role in Mimicry and Population Regulation. Memoirs of the Entomological Society of Canada, Vol. 45, 1-60. https://doi.org/10.4039/entm9745fv

[6] Alligood, K.T., Sauer, T.D. and Yorke, J.A. (1997) Chaos: An Introduction to Dynamical Systems. Springer-Verlag, New York.

https://doi.org/10.1007/978-3-642-59281-2

[7] Aziz, M.M. (2018) Stability Analysis of Mathematical Model. International Journal of Science and Research (IJSR), 7, 147-148.

[8] Aziz, M.M. and Al-Nuaimi, Z.A. (2013) Stability and Hop-Bifurcation for Diabetes Model. International Journal of Electronics Communication and Computer Engineering, 4, 805-809.

[9] Hahn, W. (1967) The Stability of Motion. Springer, New York. https://doi.org/10.1007/978-3-642-50085-5

[10] Joseph, J. and Allen, I. (1990) Feedback and Control Systems. McGraw-Hill, London.

[11] Wiggins, S. (1990) An Introduction to Applied Nonlinear Dynamics and Chaos. Springer-Verlag, New York. https://doi.org/10.1007/978-1-4757-4067-7

[12] Din, Q. (2018) Bifurcation Analysis and Chaos Control in Discrete-Time Glycolysis Models. Journal of Mathematical Chemistry, 56, 904-931. https://doi.org/10.1007/s10910-017-0839-4

[13] Xin, B. and Li, Y. (2013) Bifurcation and Chaos in a Price Game of Irrigation Water in a Coastal Irrigation District. Discrete Dynamics in Nature and Society, 2013, Article ID: 408904. https://doi.org/10.1155/2013/408904

[14] Melbourne, M., Nicol, I. and Ashwin, P. (2001) Euclidean Extensions of Dynamical Systems. Nonlinearity, 14, 275-300. https://doi.org/10.1088/0951-7715/14/2/306

[15] Falconer, I., Gottwald, G.A. and Melbourne, I. (2007) Application of the 0-1 Test for Chaos to Experimental Data. SIAM Journal on Applied Dynamical Systems, 6, 395-402. https://doi.org/10.1137/060672571

[16] Gottwald, G.A. and Melbourne, I. (2009) On the Implementation of the 0-1 Test for Chaos. SIAM Journal on Applied Dynamical Systems, 8, 129-145.

https://doi.org/10.1137/080718851

[17] Aziz, M.M. and Hamid, M.A. (2019) The Possibility of Increasing the Predictability Indices after Control of 3-D Continuous-Time System. International Conference on Computing and Information Science and Technology and Their Applications, Kirkuk, 3-5 March 2019, 1-5. https://doi.org/10.1109/ICCISTA.2019.8830650

[18] Litak, G., Syta, A., Budhraja, M. and Saha, L.M. (2009) Detection of the Chaotic Behaviour of a Bouncing Ball by the 0-1 Test. Chaos, Solitons and Fractals, 42, 1511-1517. https://doi.org/10.1016/j.chaos.2009.03.048

[19] Sun, K., Liu, X. and Zhu, C. (2010) The 0-1 Test Algorithm for Chaos and Its Applications. Chinese Physics B, 19, Article ID: 110510. https://doi.org/10.1088/1674-1056/19/11/110510

[20] Zachilas, L. and Psarianos, I.N. (2012) Examining the Chaotic Behavior in Dynami- 
cal Systems by Means of the 0-1 Test. Journal of Applied Mathematics, 2012, Article ID: 681296. https://doi.org/10.1155/2012/681296

[21] Aziz, M.M. and Merie, D.M. (2020) Stability and Adaptive Control with Synchronization of 3-D Dynamical System. Open Access Library Journal, 7, 1-18.

https://doi.org/10.4236/oalib.1106075

[22] Sundarapandian, V. and Pehlivan, I. (2012) Analysis, Control, Synchronization, and Circuit Design of a Novel Chaotic System. Mathematical and Computer Modelling, 55, 1904-1915. https://doi.org/10.1016/j.mcm.2011.11.048

[23] Murray, J.D. (1993) Mathematical Biology. Second Corrected Edition, Springer-Verlg, New York. 